\title{
An Improved Heaviside Approach to Partial Fraction Expansion and its Applications
}

\author{
Yiu-Kwong Man
}

\begin{abstract}
An improved Heaviside approach to compute the partial fraction expansions of proper rational functions is presented. This method involves simple substitutions and polynomial divisions only, without the use of differentiation or solution of a system of linear equations. Examples on its applications in some topics of engineering mathematics, such as indefinite integration, inverse Laplace transforms and differential equations, are included.
\end{abstract}

Index Terms-partial fractions, Heaviside's approach, inverse Laplace transform, differential equations.

\section{INTRODUCTION}

The problem of finding the partial fraction expansions of rational functions is often encountered in the study of integral calculus, differential equations, control theory and certain topics of applied mathematics. According to [1], there are two common methods for finding the numerators of the partial fractions. One approach is to multiply out the denominators of the rational functions involved and equate the like terms on both sides of the equality. Then, the unknown coefficients can be found by solving a system of linear equations. Another approach is to use the Heaviside's cover-up technique, which involves substitutions to determine the numerators of the partial fraction expansions with single poles, and successive differentiations to handle those with multiple poles, as pointed out in [5].

In [6], an improved Heaviside approach for computing the partial fraction expansions of proper rational functions was proposed by the author, which can be used to find the numerators of the partial fractions successively, via simple substitutions and polynomial divisions only. In this paper, a brief overview of the mathematical background of this new approach is provided. Examples on its applications in some topics of engineering mathematics, such as indefinite integration, inverse Laplace transforms and differential equations, are then provided.

\section{AN IMPROVED HEAVISIDE APPROACH}

The existence of the partial fraction expansion is based on the theorem below.

Manuscript submitted for review on 22 March 2008. This work was supported in part by the HKIEd's Research Grant on Mathematics Education.

Y. K. Man is an Assistant Professor at the Mathematics Division, Department of Mathematics, Science, Social Science and Technology, The Hong Kong Institute of Education, 10 Lo Ping Road, Tai Po, Hong Kong (e-mail: ykman@ied.edu.hk).
Theorem 2.1. Let $F$ be a constant field and $a(x)$ and $b(x)$ be polynomials in $F[x]$ such that $\operatorname{deg} a(x)<\operatorname{deg} b(x)$ and $b(x)=\left(x-\alpha_{1}\right)^{n_{1}}\left(x-\alpha_{2}\right)^{n_{2}} \cdots\left(x-\alpha_{s}\right)^{n_{s}}$, where $\alpha_{1}, \alpha_{2}, \cdots, \alpha_{s} \in F$ and $n_{1}, n_{2}, \cdots, n_{s}$ are positive integers. Then $a(x) / b(x)$ has a unique partial fraction expansion of the form

$$
\frac{a(x)}{b(x)}=\sum_{i=1}^{s}\left(\frac{a_{i, 1}}{\left(x-\alpha_{i}\right)}+\frac{a_{i, 2}}{\left(x-\alpha_{i}\right)^{2}}+\cdots+\frac{a_{i, n_{i}}}{\left(x-\alpha_{i}\right)^{n_{i}}}\right)
$$

where $a_{i, j} \in F$.

An improved Heaviside approach for computing the unknown coefficients $a_{i, j}$ is described below. The proof of its correctness can be found in [6].

Theorem 2.2. The coefficients $a_{i, j}$ in Theorem 2.1 can be determined by the following procedure:

$$
\begin{aligned}
& \text { S1: } \quad a_{i, n_{i}}=\left.\frac{a(x)}{b(x)} \cdot\left(x-\alpha_{i}\right)^{n_{i}}\right|_{x=\alpha_{i}} \\
& \mathrm{~S} 2: \quad a_{i, n_{i}-j}=\left.\left[\frac{a(x)}{b(x)}-\sum_{k=0}^{j-1} \frac{a_{i, n_{i}-k}}{\left(x-\alpha_{i}\right)^{n_{i}-k}}\right]\left(x-\alpha_{i}\right)^{n_{i}-j}\right|_{x=\alpha_{i}}
\end{aligned}
$$

where $1 \leq i \leq s$ and $1 \leq j \leq n_{i}-1$.

The substitutions involved in step S1 are called the Heaviside's cover-up technique. To find the other coefficients, we can move the partial fractions already found to the left hand side of (1) and rewrite it as a new rational function, as illustrated in S2. Simplification of this new rational function can be done by means of a single polynomial division only. Then, we can apply the whole procedure to this function recursively, until all the $a_{i, n_{-}-1}$ have been found.

Unlike the original Heaviside approach, which involves successive differentiations, namely

$$
a_{i, n_{i}-j}=\left.\frac{1}{j !} \frac{d^{j}}{d x^{j}}\left[\left(x-\alpha_{i}\right)^{n_{i}} \cdot \frac{a(x)}{b(x)}\right]\right|_{x=\alpha_{i}}
$$

where $1 \leq i \leq s$ and $0 \leq j \leq n_{i}-1$, the improved Heaviside approach is comparatively much more simple, which makes it very suitable for hand or machine calculation.

\section{APPLICATIONS}

We now illustrate how to apply this new Heaviside approach to some topics of engineering mathematics, such as indefinite integrations, inverse Laplace transforms and 
Proceedings of the World Congress on Engineering 2008 Vol II WCE 2008, July 2 - 4, 2008, London, U.K.

differential equations.

Example 3.1. Evaluate $\int\left(x^{2}+3 x\right) /(x+1)(x-1)^{2} d x$.

Solution. Let

$F(x)=\frac{x^{2}+3 x}{(x+1)(x-1)^{2}}=\frac{a}{x+1}+\frac{b}{x-1}+\frac{c}{(x-1)^{2}}$, where $a, b$,

$c$ are unknown constants to be determined. Using the cover-up technique, we have

$$
\begin{aligned}
& a=\left.(x+1) F(x)\right|_{x=-1}=\frac{1-3}{(-2)^{2}}=-\frac{1}{2}, \\
& c=\left.(x-1)^{2} F(x)\right|_{x=1}=\frac{1+3}{2}=2 .
\end{aligned}
$$

Next, we consider

$$
\frac{x^{2}+3 x}{(x+1)(x-1)^{2}}+\frac{1}{2(x+1)}-\frac{2}{(x-1)^{2}}=\frac{3}{2(x-1)} \text {. }
$$

Hence, $b=\frac{3}{2}$ and the whole partial fraction expansion is $\frac{x^{2}+3 x}{(x+1)(x-1)^{2}}=\frac{-1}{2(x+1)}+\frac{3}{2(x-1)}+\frac{2}{(x-1)^{2}}$. Integrating the partial fractions, we have

$$
\int \frac{x^{2}+3 x}{(x+1)(x-1)^{2}} d x=\frac{-1}{2} \ln |x+1|+\frac{3}{2} \ln |x-1|-\frac{2}{x-1} .
$$

The above technique is also applicable to some cases, which involve an irreducible quadratic factor in the denominator of the integrand, as illustrated in the example below. Compared with the method adopted in [2], we can see that solving a system of linear equations is not required in this new approach.

Example 3.2. Evaluate $\int\left((x+2)\left(x^{2}+3\right)\right)^{-1} d x$.

Solution. Let $F(x)=\frac{1}{(x+2)\left(x^{2}+3\right)}=\frac{a}{x+2}+\frac{b x+c}{x^{2}+3}$,

where $a, b, c$ are unknown constants to be determined. Using the cover-up technique, we have

$$
a=\left.(x+2) F(x)\right|_{x=-2}=\frac{1}{4+3}=\frac{1}{7} .
$$

Next, we consider

$$
\frac{1}{(x+2)\left(x^{2}+3\right)}-\frac{1}{7(x+2)}=\frac{4-x^{2}}{7(x+2)\left(x^{2}+3\right)}=\frac{2-x}{7\left(x^{2}+3\right)} \text {. }
$$

Hence, $b=-1 / 7, c=2 / 7$. Integrating the partial fractions, we have

$\int \frac{1}{(x+2)\left(x^{2}+3\right)} d x=\frac{1}{7} \ln |x+2|-\frac{1}{14} \ln \left|x^{2}+3\right|+\frac{2}{7 \sqrt{3}} \tan ^{-1} \frac{x}{\sqrt{3}}$.

In the study of advanced engineering mathematics, such as [3], [4], we often come across topics of inverse Laplace transform and its application to differential equations. We now illustrate how the improved Heaviside's approach can be applied to these topics.

Example 3.3. Find the inverse Laplace transform of the function $(s+1) / s\left(s^{2}+4\right)$.
Solution. Let $F(s)=\frac{s+1}{s\left(s^{2}+4\right)}=\frac{A}{s}+\frac{B s+C}{s^{2}+4}$, where $A, B$,

$C$ are unknown constants to be determined. Using the cover-up technique, we have

$$
A=\left.s F(s)\right|_{s=0}=\frac{1}{4} \text {. }
$$

Next, we consider

$$
\frac{s+1}{s\left(s^{2}+4\right)}-\frac{1}{4 s}=\frac{4-s}{4\left(s^{2}+4\right)}=\frac{1}{s^{2}+4}-\frac{s}{4\left(s^{2}+4\right)} \text {. }
$$

Hence, $B=-1 / 4, C=1$ and the inverse Laplace transform is $L^{-1}\left(\frac{s+1}{s\left(s^{2}+4\right)}\right)=\frac{1}{4}-\frac{1}{4} \cos 2 t+\frac{1}{2} \sin 2 t$.

Example 3.4. Solve the equation $y^{\prime \prime \prime}+3 y^{\prime \prime}+3 y^{\prime}+y=1$ with the initial conditions $y(0)=y^{\prime}(0)=y^{\prime \prime}(0)=0$.

Solution. Let $L[y(t)]=Y(s)$ and apply Laplace transform to both sides of the differential equation. We have

$$
\left(s^{3}+3 s^{2}+3 s+1\right) Y(s)=\frac{1}{s}
$$

i.e. $Y(s)=\frac{1}{s(s+1)^{3}}$.

Let $Y(s)=\frac{A}{s}+\frac{B}{s+1}+\frac{C}{(s+1)^{2}}+\frac{D}{(s+1)^{3}}$ where $A, B, C, D$

are unknown constants to be determined. Using the cover-up technique, we have

$$
\begin{aligned}
& A=\left.s Y(s)\right|_{s=0}=1, \\
& D=\left.(s+1)^{3} Y(s)\right|_{s=-1}=-1 .
\end{aligned}
$$

Next, we consider

$$
Y_{1}(s)=\frac{1}{s(s+1)^{3}}-\frac{1}{s}+\frac{1}{(s+1)^{3}}=\frac{-(s+2)}{(s+1)^{2}} .
$$

Using the cover-up technique again, we have

$$
\begin{aligned}
& C=\left.(s+1)^{2} Y_{1}(s)\right|_{s=-1}=-1 \\
& Y_{1}(s)+\frac{1}{(s+1)^{2}}=\frac{-(s+1)}{(s+1)^{2}}=-\frac{1}{s+1}
\end{aligned}
$$

Hence, $D=-1$ and the partial fraction expansion is $Y(s)=\frac{1}{s}-\frac{1}{s+1}-\frac{1}{(s+1)^{2}}-\frac{1}{(s+1)^{3}}$. Taking the inverse Laplace transform, we obtain $y(t)=1-e^{-t}-t e^{-t}-\frac{1}{2} t^{2} e^{-t}$.

\section{CONCLUDING REMARKS}

An improved Heaviside approach to compute the partial fraction expansions of proper rational functions has been introduced in this paper. This method can be used to find the unknown numerators of the partial fractions successively, via simple substitutions and polynomial divisions only, without the use of differentiation or solution of a system of linear equations. We have also illustrated its applications in some topics of engineering mathematics. Due to its simplicity and effectiveness, it can be introduced to undergraduate students or instructors who are studying or teaching engineering mathematics, as an alternative to those classical techniques found in the engineering mathematics textbooks. 
Proceedings of the World Congress on Engineering 2008 Vol II WCE 2008, July 2 - 4, 2008, London, U.K.

\section{REFERENCES}

[1] L.B. Norman, Discrete Mathematics, 2nd ed. New York: Oxford University Press, 1990, pp. 403-407.

[2] J. Mikusinski \& P. Mikusinski, An Introduction to Analysis. New York: John Wiley \& Sons, 1993, pp. 184-186.

[3] E. Kreyszig, Advanced Engineering Mathematics. New York: John Wiley \& Sons, 1999, pp. 284-288.

[4] D. W. Jordan \& P. Smith, Mathematical Techniques: An Introduction for the Engineering, Physical and Mathematical Sciences. Oxford: Oxford University Press, 2002, pp. 455-464.

[5] S. H. Hou \& S.H. Edwin Hou, "On partial fraction expansion with multiple poles,” Int. J. of Math. Edu in Sci. \& Tech., vol. 35, 2004, pp. 782-791.

[6] Y. K. Man, "A simple algorithm for computing partial fraction expansions with multiple poles,” Int. J. of Math. Edu in Sci. \& Tech., vol. 38, 2007, pp. 247-251. 